\title{
Observation of the in vitro production of male gametes of the human malaria parasite Plasmodium falciparum through the process of exflagellation
}

\author{
Martin Looker and Andrew W Taylor-Robinson* \\ ${ }^{1}$ Institute of Molecular \& Cellular Biology, University of Leeds, Leeds LS2 9JT, UK \\ ${ }^{2}$ School of Health, Medical \& Applied Sciences, Central Queensland University, Brisbane, QLD 4000, Australia
}

\section{Case report}

Exflagellation is the term given to the production of male gametes by sporozoans, in particular the Plasmodium parasite that causes malaria in a wide range of vertebrates including humans. In the malaria life cycle this key event occurs naturally within the midgut of a female Anopheles mosquito a few minutes after it takes a blood meal infected with Plasmodium gametocytes [1]. This phenomenon also happens spontaneously in vitro and thus may be observed in cultures of fresh parasitized blood under the light microscope (1000 x magnification under oil immersion) [2].

We report the rare observation in vitro of male (micro)gametogenesis by the major human malaria parasite P. falciparum (Figure 1). While this process may be recorded by video, by which its explosively energetic nature can readily be appreciated, it is not straightforward to capture by still photography because the parasite oscillates vigorously and thus moves continually in and out of the microscope's two-dimensional plane of focus.

This photograph is an unusually clear and detailed image of this metabolically dynamic and visually striking event. The nucleus of the parent male gametocyte divides rapidly three times to form eight daughter nuclei, each of which has an associated axoneme. This nuclear material moves to the surface of the gametocyte to be extruded into peripheral cytoplasmic processes as the spindle-shaped microgametes

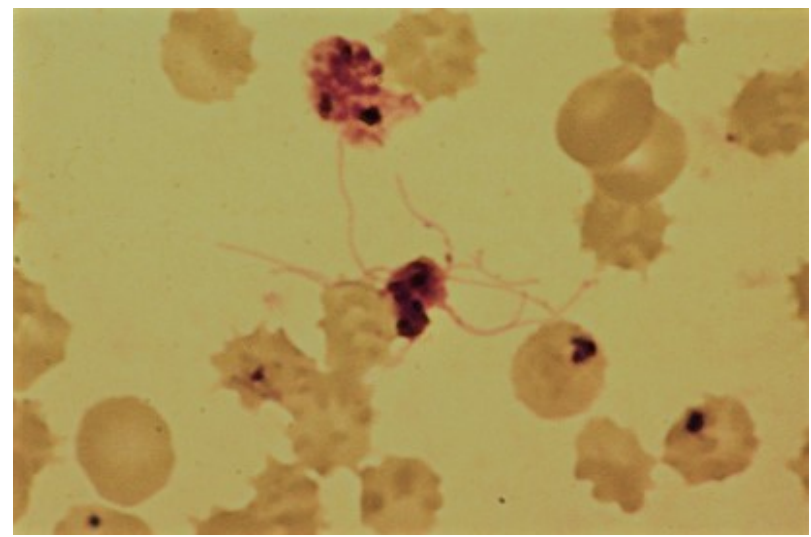

Figure 1. Male (micro)gametocyte of the human malaria parasite Plasmodium falciparum, cloned line 3D7A, cultivated in vitro in group $\mathrm{O}$, rhesus group-positive human erythrocytes. Exflagellation (arrowed) - extrusion of eight motile, flagella-like microgametes with vigorous movement. Blood film, wet mount, $1000 \mathrm{x}$ magnification under oil immersion with visibly identifiable flagella. Gametogenesis may be viewed typically between 10 to 25 minutes after removal of cultures of parasitized blood from an incubator maintained at $37^{\circ} \mathrm{C}$. The gas mixture of choice is $1 \%$ oxygen, $3 \%$ carbon dioxide and $96 \%$ nitrogen.

Under in vitro conditions exflagellation is controlled solely by the drop in air temperature from $37^{\circ} \mathrm{C}$ at which $P$. falciparumcontaining human blood cultures are routinely maintained to the ambient laboratory temperature (of around $22-24{ }^{\circ} \mathrm{C}$ ), the $\mathrm{pH}$ rise this brings being mediated by a fall in carbon dioxide tension as the blood equilibrates with the atmosphere [2]. This cascade mimics the environmental changes that occur to the gametocytes in the gut of the mosquito which exhibits a $\mathrm{pH}$ of approximately 7.8 as compared to a $\mathrm{pH}$ of 7.4 in human blood.

The visual recording of microgametogenesis in vitro is a reliable indicator that oocysts and sporozoites will most likely be produced in vivo following membrane-feeding of a batch of the same blood culture to adult female mosquitoes. Typically, in a successfully fed mosquito oocysts may be observed on the outer midgut wall 10 days post infection, while sporozoites may be dissected from the salivary glands a further 7 days later [3]. These sexual stages of the parasite, which occur only within the mosquito host, are required in order to research vector infectivity.

The artificial triggering of male gametogenesis is therefore crucial for investigating the sexual stages of $P$. falciparum. This is a prerequisite to the successful infectivity of $P$. falciparum for the insectary standard species of mosquito, A. stephensi, which may be routinely achieved using the protocol described by ourselves previously [4]. This provides a powerful tool to enable the conduct of a wide range of molecular and cellular studies of the sexual stages of $P$. falciparum in vivo.

\section{Discussion}

Despite a recent reduction in the annual toll of morbidity and mortality the mosquito-borne infectious disease malaria remains

${ }^{\star}$ Correspondence to: AW Taylor-Robinson, School of Health, Medical \& Applied Sciences, Central Queensland University, 160 Ann Street, Brisbane, QLD 4000, Australia, Tel: +61 73295 1185, E-mail: a.taylor-robinson@cqu.edu.au

Key words: malaria, Plasmodium falciparum, cultivation, gametocyte, gamete, exflagellation

Received: June 13, 2018; Accepted: June 28, 2018; Published: June 30, 2018 
a major global public health concern, with an incidence of several hundred million clinical cases and up to 3 million deaths each year [5].

While it has rendered ineffectual many strategies for infection control, increasing resistance to drugs of the aetiological agent, protozoan Plasmodium parasites, and to insecticides of the vector, Anopheles mosquitoes, is demanding novel perspectives to counter both the transmission of infection and burden of the disease [6]. Of the six species of malaria that are known to infect humans, $P$. falciparum is by far the deadliest and therefore the one towards which most control measures are focused.

A principal reason for the modest success of one strategy, antimalaria vaccine design, is our inadequate understanding of the developmental processes that operate, and antigens expressed, during the differentiation of parasite stages [7], including those within the Anopheles vector. Immunity to sexual stages of malaria, termed transmission-blocking immunity, may operate against gametocytes in the vertebrate host and against gametes in the midgut and ookinetes, the motile form of the Plasmodium parasite that traverses the mosquito midgut epithelium to initiate sporogony [8].

In order to facilitate detailed molecular, biochemical and immunological studies of Plasmodium parasite progression within the Anopheles mosquito, it is necessary to produce sufficient numbers of fully mature oocysts and sporozoites [9]. This requires the in vitro cultivation of gametocytes and the laboratory-manipulated induction of gametogenesis prior to a mosquito blood feed. The exflagellation event shown in Figure 1 is a strong predictor of a successful outcome to this sexual stage parasite harvesting process.

It should be noted that $P$. falciparum is a highly dangerous human pathogen that is classified internationally as requiring biosafety level 3 containment [10]. Hence, when maintaining this parasite in human blood in vitro all appropriate health and safety measures must be performed in adherence to local regulations and institutional guidelines. Any isolate of $P$. falciparum that is used routinely for laboratory-based research purposes should be susceptible to standard antimalarial drugs.

\section{Acknowledgement}

This work received financial support from the Wellcome Trust, the Royal Society and the University of Leeds.

\section{Disclosure}

No conflicts of interest.

\section{References}

1. Dixon MW, Thompson J, Gardiner DL, Trenholme KR (2008) Sex in Plasmodium: A sign of commitment. Trends Parasitol 24: 168-175. [Crossref]

2. Carter R, Nijhout MM (1977) Control of gamete formation (exflagellation) in malaria parasites. Science 195: 407-409. [Crossref]

3. Looker M, Taylor-Robinson AW (2014) A protocol for a highly consistent, high level production of Plasmodium falciparum oocysts and sporozoites. Adv Biosci Biotechnol 5: $985-993$

4. Looker M, Taylor-Robinson AW (2013) Cultivation of Plasmodium falciparum gametocytes for mosquito infectivity studies. In: K Moll, A Kaneko, A Scherf, M Wahlgren, eds. Methods in Malaria Research, 6th edn, pp. 138-141. Glasgow, UK: EVIMalaR.

5. Murray CJ, Rosenfeld LC, Lim SS, Andrews KG, Foreman KJ, et al. (2012) Global malaria mortality between 1980 and 2010: a systematic analysis. Lancet 379: 413431.

6. Breman JG, Brandling-Bennett AD (2011) The challenge of malaria eradication in the twenty-first century: research linked to operations is the key. Vaccine 29: D97-D103.

7. Taylor-Robinson AW (2010) Regulation of immunity to Plasmodium: implications from mouse models for blood stage malaria vaccine design. Exp Parasitol 126: 406414. [Crossref]

8. Angrisano F, Tan YH, Sturm A, McFadden GI, Baum J (2012) Malaria parasite colonisation of the mosquito midgut - placing the Plasmodium ookinete centre stage. Int J Parasitol 42: 519-527. [Crossref]

9. Matuschewski K (2006) Getting infectious: formation and maturation of Plasmodium sporozoites in the anopheles vector. Cell Microbiol 8: 1547-1556. [Crossref]

10. Centers for Disease Control and Prevention - National Institutes of Health (2009) Biosafety in Microbiological and Biomedical Laboratories, 5th edn. US Department of Health and Human Services, Bethesda, MD, USA. Available at: http://www.cdc.gov/ biosafety/publications/bmbl5/BMBL.pdf [Accessed 18 July 2018].

Copyright: (C2018 Looker M. This is an open-access article distributed under the terms of the Creative Commons Attribution License, which permits unrestricted use, distribution, and reproduction in any medium, provided the original author and source are credited. 